\title{
CARACTERIZAÇÃO DA PRODUÇÃO FLORESTAL NÃO-MADEIREIRA NA AMAZÔNIA LEGAL:SUBSÍDIOS PARA GESTÃO ECONÔMICA E AMBIENTAL
}

\author{
Aline Silva de Oliveira* \\ Ricardo Alexandrino Garcia
}

\section{Resumo}

Tradicionalmente as análises econômicas realizadas na Amazônia Legal ou evidenciam a exploração de madeira ou o uso da terra para agropecúaria e comumente negligenciam o valordos produtos florestais não-madeireiros (PFNMs) extraídos da floresta. Tendo em vista esse processo, foi desenvolvido nesse trabalho um modelo estatístico de análise multivariada FUZZY Cluster para verificar a produtividade de PFNMs por município na Amazônia Legal, com o objetivo de fornecer informações colaborativas à gestão econômica e ambiental dessa atividade na região. A produtividade foi calculada através dos dados de quantidade produzida e valor de produção levantados pelo IBEG (2009) para os anos de 2004 a 2008. Uma análise espacial dos clusters gerados foi realizada para propor uma nova regionalização econômica em torno da produção de PFNMs. Para melhor compreensão dos resultados e da dinâmica de produção dos PFNMs na Amazônia foram incluídas nas análises, variáveis importantes para o entendimento da lógica mercantil e rentabilidade desses produtos, como os princípios de manejo sustentável, mercado, beneficiamento, escoamento da produção e aspectos socioeconômicos e ambientais.

Palavras-chave:Produtos Florestais não madeireiros (PFMNs), Fuzzy cluster, Amazônia Legal.

\section{Abstract}

Traditionally, economic analysis conducted in the Amazon show only the timber or the use of land for farming and ranching, neglecting the value of non-timber forest products. In order to fill in part of this gap, a statistical model of multivariate analysis Fuzzy Cluster was developed to verify the productivity of NTFPs by municipality in the Brazilian Amazon, aiming to provide collaborative information management and economic and environmental activity in this region. The productivity was calculated using amount produced and production value data from the IBGE (2009) for the years 2004 to 2008. A spatial analysis of the clusters was done in order to propose a new economic regionalization around the production of NTFPs. To better understand the results and dynamics of NTFPs production in the Amazon important variables were included in the analysis to understand the logic of commerce and profitability of these products, such as socioeconomic and environmental aspects, principles of sustainable management, marketing, product processing and transport.Key words: Non-timber Forest Products, Fuzzy cluster, Amazon

\footnotetext{
*Universidade Federal de Minas Gerais - UFMG/IGC Belo Horizonte - MG, Brasil. alinesilva.ufmg@gmail.com

-Universidade Federal de Minas Gerais - UFMG/IGC Belo Horizonte - MG, Brasil. alexandrinogarcia@gmail.com 


\section{1- INTRODUÇÃO}

Os recursos florestais não- madeireiros (PFNMs) ${ }^{1}$ consistem, atualmente, em uma dasprincipais fontes de renda de cerca de 500 mil famílias que vivem da extração florestal naAmazônia. Produtos como frutos, óleos, essências, amêndoas, fibras, plantas medicinais e outras de grande ocorrência nesse tipo de floresta ${ }^{2}$, constituem-se em uma oportunidade promissora para o incremento da renda dos extrativistas, seja pela exploração em manejo ou em cultivos familiares (Embrapa, 2000).

Os produtos naturais atendem não só o autoconsumo dessas famílias, mais também osmercados interno e externo, com tendência a mercantilização crescente e âmbito geográfico cada vez maior. Estudos mostram que, além do potencial de ampliação dos PFNMs, a atividade pode proporcionar maior engajamento das populações locais, que passam a ter além de um importante componente de subsistência, novas alternativas de trabalho (Fiedler, 2008). Segundo Brito (2003) há indicações de que a geração de empregos através de PFNM é de 5 a 15 vezes maior do que no processo de exploração madeireira.

Os produtos naturais atendem não só o autoconsumo dessas famílias, mais também os mercados interno e externo, com tendência a mercantilização crescente e âmbito geográfico cada vez maior. Estudos mostram que, além do potencial de ampliação dos PFNMs, a atividade pode proporcionar maior engajamento das populações locais, que passam a ter alémde um importante componente de subsistência, novas alternativas de trabalho (Fiedler, 2008).Segundo Brito (2003) há indicações de que a geração de empregos através de PFNM é de 5 a15 vezes maior do que no processo de exploração madeireira. Além disso, a extração desse tipo de produto tem se constituído com uma atividade fundamental por ser provedora de recursos naturais, uma vez que permite valorizar a florestaque é preservada em pé, pois não implica na derrubada das árvores matrizes. Ao contrário daexploração madeireira que contribui com a disseminação das espécies de maior valor comercial, o que compromete seu aproveitamento futuro.

Bentes (2005) afirma que as indústrias madeireiras pagam um preço muito baixo pelo metro cúbico da madeira em pé que varia em média de 2 a 8 reais, sendo essa baixa valoraçãouma das razões que colaboram para a exploração florestal predatória. Uma maneira possível de amenizar esse processo na Amazônia e diminuir o desmatamento em massa seria justamente, estimular o mercado e a utilização dos produtos florestais nao- madeireiros, queaté o momento,

\footnotetext{
1“'São produtos florestais não-lenhosos de origem vegetal e animal, bem como serviços sociais e ambientais, como reservas extrativistas, sequestro de carbono, conservação genética e outros benefícios oriundos da manutenção da floresta" (Embrapa, 2000).

${ }^{2}$ Florestas tropicais úmidas que possuem vegetação exuberante, densa e com altas árvores. O clima quente eúmido fornece muita chuva durante todo ano. Essas florestas são encontradas na África, Ásia, América Central eAmérica do Su l, sendo a maior floresta tropical ú mida do mundo a Amazônica (Soares \& Pessoa (2008).
} 
com raras exceções como a castanha-do-brasil, ainda não dispõem de um mercado real, sendo necessária a construção de políticas nacionais de criação e expansão dedemandas para estes produtos (Toniniet al, 2008). Seria uma forma de converter parte da obtenção de renda para a comercialização de PFNMs.

Ademais, a elaboração de políticas específicas para essa atividade, bem como o investimento em tecnologia para o aprimoramento da produção de PFNMs, colaboraria m coma ruptura da atividade a partir de moldes rudimentares, cujos métodos tradicionais de extraçãonormalmente geram um grande desperdício de matéria prima, qualidade e um baixo preço pago por estes produtos.

Aliada a essas perspectivas, instituições governamentais e não-governamentais atuantes na Amazônia, enfatizam a importância de um acompanhamento técnico apropriadopara a extração dos recursos naturais e um planejamento estratégico para utilização sustentável dos PFNMs. As propostas buscam otimizar essa atividade priorizando a conservação da floresta e atendendo expectativas como geração de renda e melhoria da qualidade de vida.

Tendo em vista, portanto, que em um cenário mais atual, as mudanças causadas por pressões ambientalistas e econômicas na Amazônia catalisaram o interesse da ciência e de governos contemporâneos também para os produtos florestais não madeireiros (Fiedler et al,2008), esse trabalho se constitui em mais um estudo colaborativo e complementar às ferramentas de gestão econômica e ambiental para região.

$\mathrm{Na}$ perspectiva de elaborar uma análise econômica diferenciada da Amazônia, já que tradicionalmente esse tipo de análise para região evidencia a exploração madeireira ou uso daterra para agricultura e pecuária e negligencia o valor dos produtos florestais não- madeireirosextraídos da floresta (Castellani, 2002), foi realizado nesse trabalho um modelo estatístico deanálise multivariada FUZZY Cluster, para verificar a produtividade de PFNMs por municípiona Amazônia Legal e a partir de uma análise espacial propor uma nova regionalização econômica em torno da produção de PFNMs. Foram também incluídas nas análises, variáveisimportantes para o entendimento da lógica mercantil, rentabilidade e produtividades desses produtos, como, princípios de manejo sustentável, mercado, beneficiamento e escoamento daprodução, e aspectos socioeconômicos e ambientais.

\section{2 - MATERIAIS E METODOS}

Considerando que PFNMs podem propiciar tanto recursos provenientes da flora quanto da fauna e variados outros serviços ecológicos. É importante salientar que nesse trabalho foram 
analisados somente PFNMs originários do extrativismo vegetal de diferentesqualidades (alimentícios, aromáticos, borracha, ceras, óleos, fibras e tanantes).

Para a realização da análise de produtividade foram coletados os dados de "Quantidade de PFNMs Produzida" (Toneladas) e "Valor de Produção" (Reais) por municípiopara os anos de 2004 a 2008. Os municípios totalizam 760 conforme a amostra fornecida pelo Instituto Brasileiro de Geografia e Estatística - IBGE para a Amazônia Legal. Mais especificamente os dados foram buscados no banco de dados agregados PAM-2009 (produçãoagrícola municipal) do Sistema do IBGE de Recuperação Automática - SIDRA.

Tabela 01: Produtos Florestais não madeireiros produzidos na Amazônia Legal

Do total de 40 produtos florestais não madeireiros caracterizados no conjunto de dados, foram selecionados os 21 prEAMAs (Tabela 01) que apresentapam valores de quantidade Açaí Alimenticio

\begin{tabular}{|c|c|}
\hline rodecasanarades municipios da & Amazoniatifieghtício \\
\hline Castanha-do-pará & Alimentício \\
\hline Palmito & Alimenticio \\
\hline Umbu & Alimentício \\
\hline Jaborandi & Aromático \\
\hline Hevea (látex coagulado) & Borracha \\
\hline Hevea (látex liquido) & Borracha \\
\hline Carnaúba & Cera \\
\hline Buriti & Fibra \\
\hline Carnaúba & Fibra \\
\hline Piaçava & Fibra \\
\hline Maçaranduba & Goma não elástica \\
\hline Sorva & Goma não elástica \\
\hline Babaçu & Oleaginoso \\
\hline Copaiba & Oleaginoso \\
\hline Cumaru & Oleaginoso \\
\hline Licuri & Oleaginoso \\
\hline Pequi & Oleaginoso \\
\hline Tucum & Oleaginoso \\
\hline Angico & Tanante \\
\hline
\end{tabular}

Fonte: Produção Agrícola Municipal - IBGE. 2009.

Para gerar o modelo estatístico FUZZY cluster foi preciso inicialmente calcular a média dos dados de quantidade produzida e valor de produção dos PFNMs entre os anos selecionados e calcular a produtividade $(\mathrm{R} \$ / \mathrm{Kg})$ a partir dessas médias. Posteriormente, os dados de produtividade foram inseridos no software SPSS 12.0 para a elaboração da análise multivariada 
através do método FANNY - Fuzzy Cluster Analysis e no software ArcMap. 9.3para análise espacial.

\subsection{FUZZY Clusters}

Os métodos de aglomeração (clustering) são procedimentos estatísticos que classificam um conjunto de dados finito e multivariado em grupos restritos e homogêneos internamente, permitindo o desenvolvimento de tipologias analíticas desse conjunto (Simões,2004).

Dentre esses métodos de classificação, destacam-se aqueles que se utilizam dos conceitos dos conjuntos clássicos (crisp sets) e dos conjuntos nebulosos (fuzzy sets). $\mathrm{Na}$ teoriaclássica, os conjuntos são ditos "crisp" , uma vez que o dado elemento do universo em discurso "pertence" ou "não pertence" ao referido conjunto, há somente duas situações possíveis. Na teoria dos conjuntos nebulosos existe um grau de pertinência de cada elementoa um determinado conjunto, de modo a tornar possível a representação matemática de conceitos vagos e imprecisos (Ribeiro \& Garcia, 2005). Exemplos são conceitos do tipo “saudável”, “deprimido” e “alto”, considerados nebulosos, pois não deveriam ser definidos demaneira extrema como seria na teoria clássica, ou seja, não existem no mundo apenas pessoasaltas (0) e não altas (1) existem graus de certeza (ou incerteza) para que pessoas possam ser classificadas como altas ou não altas, sendo esse grau classificado em um intervalo de $[0,1]$. Oconceito formal de conjunto nebuloso, fundamentado da Lógica Difusa ${ }^{3}$, foi introduzido porZadeh em 1965.

Um conjunto nebuloso é caracterizado por uma função característica de pertinência que mapeia os graus de pertinência dos elementos de um domínio X para o intervalo $[0,1]$.

Isto significa:

Sejam:

A: Conjunto Nebuloso.

X: Domínio.

$A: X_{-}[0,1]$.

ou , em termos de sua função de pertinência:

$\mathrm{A}=\{\mathrm{x} \hat{\mathrm{I} X} \mid \mathrm{A}(\mathrm{x})=1\}$, porém, neste caso:

\footnotetext{
${ }^{3} \mathrm{Na}$ Lógica Difusa ou Fuz:yy, uma premissa varia em grau de verdade de 0 a 1 , o que leva a ser parcialmente verdadeira ou parcialmente falsa. 
$\mathrm{A}(\mathrm{x})$ : $\mathrm{X}_{-}[0,1]$, ou seja, a função $\mathrm{A}(\mathrm{x})$ é a Função de Pertinência que expressa o quanto um dado elemento x pertence ao Conjunto A num intervalo de variação onde os graus de pertinência podem assumir valores de 0 a 1 (inclusive).

O diferencial da Lógica Fuzzy deriva, portanto, da sua habilidade em inferir conclusões e gerar respostas baseadas em informações vagas, ambíguas e qualitativamente incompletas e imprecisas, por isso se constitui como um método poderoso para a solução de problemas apresentando uma vasta aplicabilidade, especialmente, nas áreas de controle e tomada de decisão.

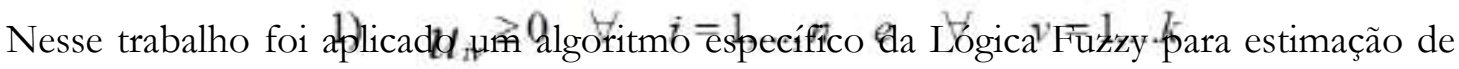
clusters, denominado FUNNY-fuzzyanalysis. Kaufman \&Rousseeuw (1990), afirmam que o método FANNY comparade $)^{a}$ oषtros métpdos de estimação fuzzy cluster, como o C-Means, por exemplo, "tem a vantagem de aéitar matrizes de dissimilaridade em todas as métricas para conjuntos contínuos e ser mais robusto que os demais" (Simões, 2004). Dessa forma para cada elemento i e cada cluster v há uma pertinência uiv que indica quão fortemente i pertence a v, se satisfeitas as condições:

$$
f=\sum_{v=1}^{k} \frac{\sum_{i, j-1}^{n} u_{N}^{2} u_{j}^{2} d(i, j)}{2 \sum^{n} u^{2}}
$$

Segundo Ribeiro \& Garcia (2005) o metod, $2 \sum_{1} \boldsymbol{u}_{\mathrm{ANN}}^{2}$ clusters, sendo essas associações definidas através de processos iterativos, buscando-se a minimização da função $\mathrm{f}$ :

Assim como diversos outros modelos e técnicas, a aplicação do FANNY - fuzzyanalysis só terá sentido se acompanhado, e precedido, de profundo conhecimento dos elementos que se deseja modelar e de fundamentos teóricos consistentes. 


\section{3 - CARACTERIZAÇÃO DA ÁREA DE ESTUDO}

A Amazônia Legal foi instituída através de dispositivo de lei para fins de planejamento econômico e ambiental da região amazônica. Engloba os Estados da macrorregião Norte (Acre, Amazonas, Amapá, Pará, Rondônia, Roraima e Tocantins), mais oEstado do Mato Grosso (macrorregião Centro-Oeste), parte do Maranhão, a oeste do meridiano de $44^{\circ}$ (macrorregião Nordeste) e cinco municípios de Goiás cortados pelo paralelo13 ${ }^{\circ}$, totalizando 761 municípios (IBGE, 2005). A área da região perfaz uma superfície aproximada de 5 milhões de km2, o que corresponde a cerca de $60 \%$ do território brasileiro.

O bioma amazônico abriga um terço das florestas tropicais e mais de $20 \%$ das espécies

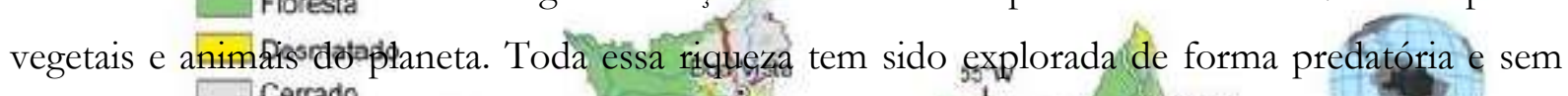
planejamento, com pouco ou genhum beńefício econômico e socials para a populaçãolocal. Conforme os dados do PRODES/INPE que monitora o desmatametitóna na região desde1998, até o momento, cerca de $18 \%$ da floresta amazônica brasileira já foi exterminafda éos os resultados

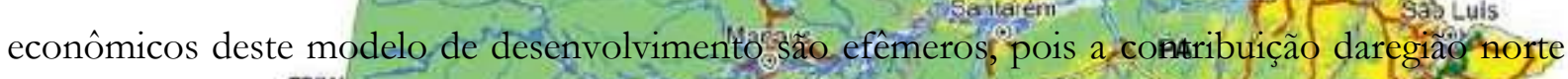
para o $\mathrm{PIB}_{5^{\prime} \mathrm{S}}$ pais é de apenas 5\%, com uma renda per capta que chega a $65 \%$ damédia hacional (Tonini et al, 2008).

AM

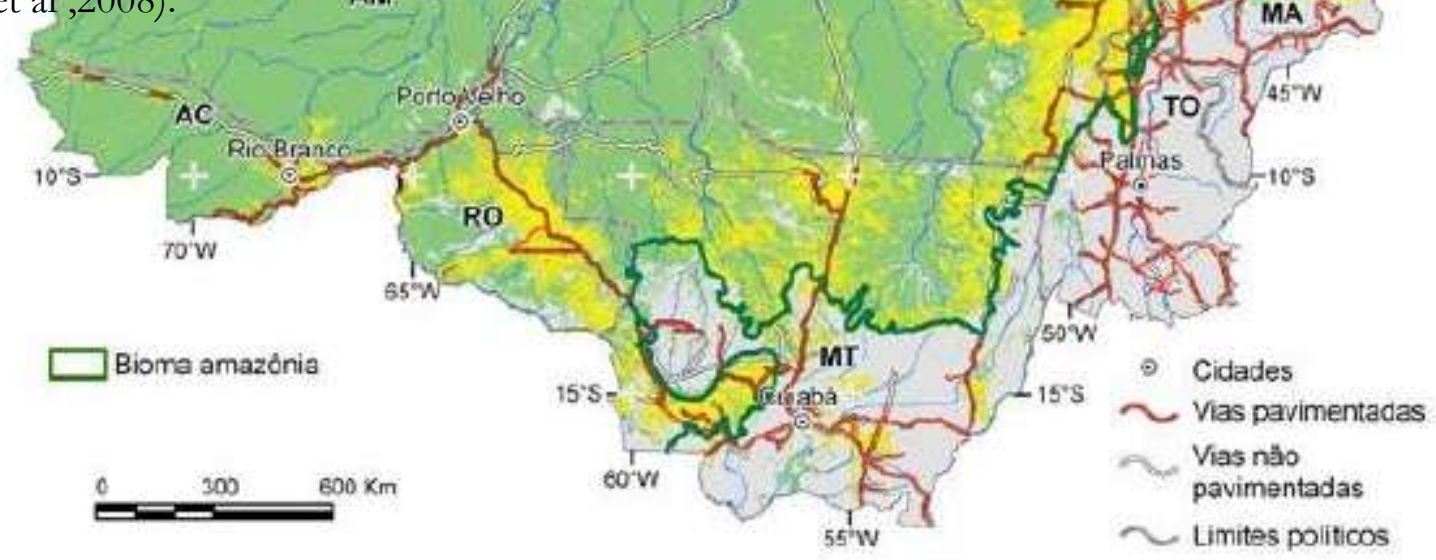




\section{4 - RESULTADOS}

A aplicação do algoritmo FANNY - fuzzy cluster analysis gerou inicialmente três clusters de municípios (agrupados conforme a suas produtividades em relação aos produtos florestais não madeireiros) com seus respectivos graus de pertinência para cada um dos grupos. Foi observado que 65\% dos municípios apresentaram valores de pertinência muito aproximados para dois dos grupos ou para todos eles, como por exemplo, o município de Augusto Corrêa - PA que apresentou grau de pertinência da produtividade igual a 0,36 para oprimeiro grupo de PNFMs, grau de 0,30 para o segundo grupo e 0,34 para o terceiro. O quetorna difícil sua caracterização ou classificação em relação a produtividade dos PNFMs, já que esse município demonstra pertencer quase que com a mesma grandeza a todos os gruposoriginados pelo FANNY-analysis. Dessa forma foram gerados subclusters a partir da somados graus de pertinência dos clusters originais, em que foram aplicados algoritmos lógicos que estabeleceram um limiar de $75 \%$ de pertencimento para esses novos grupos. Em outras palavras os novos clusters possuem somente elementos que os constituem com $75 \%$ de pertinência.

O novo agrupamento realizado a partir dos três primeiros clusters gerados pelo algoritmo FUNNY, originou 4 novos clusters, conforme demonstrados na tabela 02. O primeiro cluster, denominado C1(sem produção), constitui os municípios que não produzemnenhum dos 21 PFNMs selecionados, o segundo cluster C2 constitui os municípios que produzem predominantemente produtos "alimentícios e borrachas", o cluster C3 os municípios que produzem predominantemente "amêndoas e tanante angico" e o cluster C4constitui os municípios que produzem todos os 21 PFNMs selecionados, independente da predominância.

A partir do Mapa 02 nota-se que os municípios que constituem o cluster C1, ou seja, não produzem nenhum tipo de PFNMs, pertencem em sua maioria aos estados do Mato Grosso, Tocantins, Maranhão e Pará. Obviamente porque esses Estados possuem as maioresáreas devastados do bioma (Mapa 01) e, por conseguinte as maiores taxas de desmatamento da região amazônica, onde a floresta nativa foi em grande parte substituída pela produção demonoculturas, como a soja, e pela pastagem para a criação de gado. Outro fator determinanteé a predominância do bioma do cerrado nesses Estados, o qual sua biota natural não forneceos produtos aqui estudados, que são típicos de florestas tropicais úmidas. 
Tabela 02: Clusters de municipios da Amazōnia Legal classificados conforme a media da produtividade dos PFNMs entre os anos de 2004 a 2008

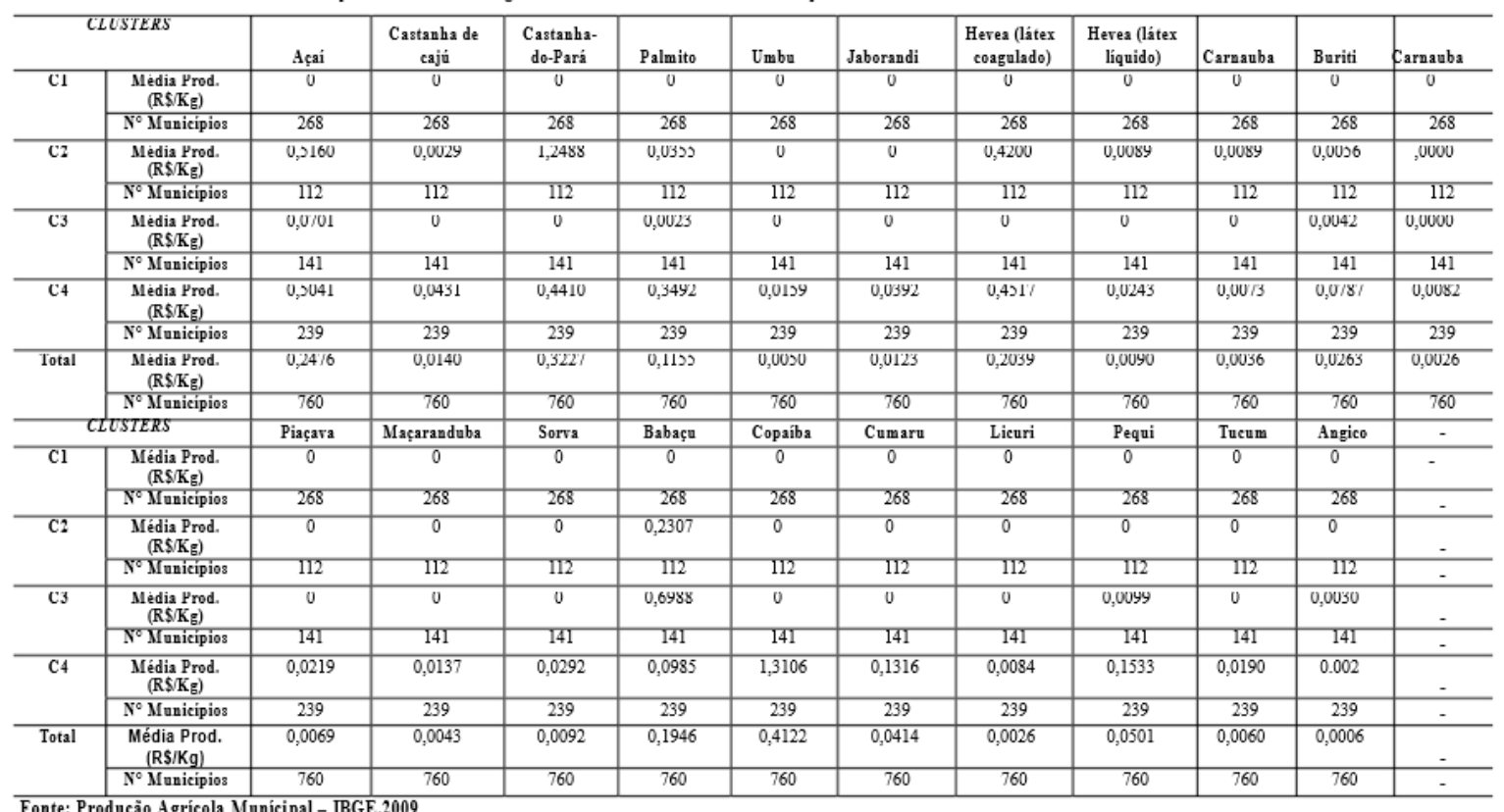

Fonte: Produção Agrícola Munícipal - IBGE.2009 Há também no nordeste de Roraima đờins municípios pertencentes ao cluster C1, sendo a ausência de produção conseqüência tamberm da maior incidência do bioma cerrado ce do do desmatamento. Os municípios da região oeste desse mesmo ${ }^{55^{\circ} \mathrm{W}}$ Estado também não aprountaram produtividade. OS motivgs provavelmente estãonassociados ao fato Adel que essa Estradias é praticamente toin ocupada por indígenas RP anomami que recebeu do govérno- $95 \%$ do território da Floresta nacional de Roraima (FLONA de Roraima) esos $5 \%$ restantes da Flona ainged, foi invadida por dois assentamentos, Samaúma Manais Yova criados pelo INCRA em 1996. Con isso $70 \mathrm{w}$ (5,20\% restaram somente cerca de 138.000 ha $(5,20 \%)$, como unidade de uso sustentável. Dessa forma d

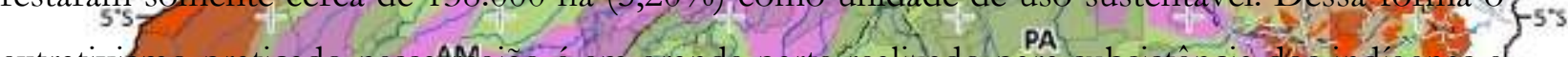
extrativismo praticado nessaAfQgião é em grande parte realizado para subsistência dós indígtanas es das pequenas famílias assentadas, possivelmente a pouca valoração comercial dos produto que se comercializadôs são para complementacão de renda familiar, tornam os municípios da região ausentes de produtividade? $\underbrace{10 \mathrm{~s}}_{70^{\circ} \mathrm{W}} \mathrm{RO}$

Áreas naturais protegidas

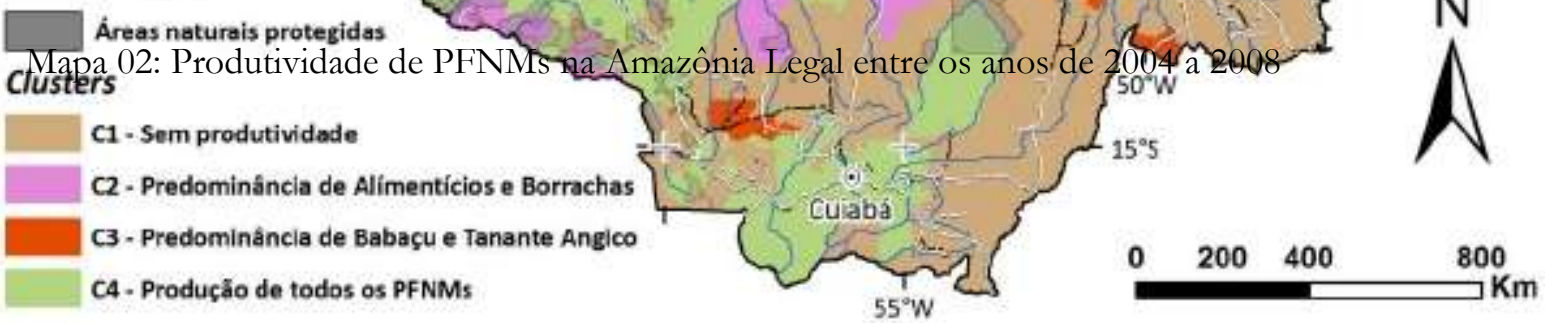


Fonte: Produção Agrícola Munícipal - IBGE.2009

A maior produtividade dos PFNMs dos tipos alimentícios e borrachas característica dos municípios constituintes do cluster C2 se devem a produção do açaí, castanha-do-pará elátex coagulado. Por essas características o grupo C2 merece destaque, já que apresenta alta produtividade dos três principais produtos que hoje são os mais valorizados, se tratando de PFNMs, no mercado interno e externo. $\mathrm{O}$ açaí, por exemplo, tem apresentando um crescimento exorbitante de sua produção nos últimos tempos. Segundo Castro (2007) a novaconjuntura econômica do mercado do açai, tanto em nível local quanto nacional e internacional, em que se tem uma crescente demanda, fizeram com que somente em 2004, o Pará ${ }^{4}$, que possui $25 \%$ dos municípios do cluster C2, exportasse cerca de 3 milhões e quinhentos dólares em polpa para os EUA, Europa e Japão. Já a castanha do Brasil foi um produto que movimentou, somente em 2006, 43,9 milhões de reais, sendo a maior parte da produção frequentemente exportada para Alemanha, Inglaterra e EUA. E por último, em setratando da produção de borracha na Amazônia, destacam-se as suas demandas no mercadointernacional como matéria prima bruta e o seu beneficiamento regional direcionado a produção de preservativos, couro vegetal, luvas e pneus. Atualmente os lucros oriundos da produção de borracha estão mais direcionados para a mercantilização interna e regional, umavez que a Amazônia que já representou o maior produtor

\footnotetext{
${ }^{4}$ O Estado do Pará é o maior produtor mundial dessa fruta, que segundo Santana (2006) gerou, no ano de 2004, o valor de $\mathrm{R} \$ 317,83$ milhões, empregou diretamente 5.650 pessoas e ocupou em algumas fases da cultura (plantio ou manejo, tratos culturais, colheita e comercialização) 150 mil pessoas, envolvendo 45 mil famílias.
} 
de borracha no mundo perdeu essemonopólio para Tailândia e Indonésia que são os atuais responsáveis pela produção mundial.

Uma análise do mapa 02 permite inferir que os municípios que formam o cluster C2 estão distribuídos por toda a Amazônia Legal com uma pequena concentração na região centro-oeste do bioma. Pertencem a esse grupo os municípios do estado do Acre, Rio Branco, Brasiléia e Xapurí, onde está localizada uma das maiores reservas extrativistas da Amazônia, a RESEX Chico Mendes. Conforme os dados do IBGE (2006), o Acre concentrou 35, 5\% da produção de castanha-do-brasil e somente o município de Rio Branco deteve 9,9\% do total da produção. É também na reserva Chico Mendes que grande parte da borracha amazônica é extraída, assim como na maioria dos municípios da mesorregião do vale do Acre, todos pertencentes ao cluster C2. Segundo dados do Ministério da Saúde de 2008, o Governo do Acre assinou um convênio com esse mesmo ministério de $\mathrm{R} \$ 22$ milhões para produção de 100 milhões de preservativos masculinos na Natex, fábrica instalada no município de Xapuri. A assinatura marcou, oficialmente, a inauguração da indústria. Outro Estado que se destaca pela produção de borracha é o Amazonas. Os seringais nativos desse Estado geram algo emtorno de mil toneladas do produto, o que responde a pouco mais de 1\% da produção brasileira de borracha. Dentre os municípios produtores destacam-se São Sebastião do Uatumã e Itapiranga, ambos vizinhos da capital Manaus.

A produção desses três PFNMs nos municípios pertencentes ao cluster C2 são otimizados não só pela disponibilidade das espécies nativas, mas por possuírem infra-estrutura necessária, como as vias de escoamento, BR 364 que atravessa todo Acre, as rodovias Belém Pará e Manaus - Porto Velho, a grande densidade de rios, com destaque para o rio Amazonas que corta todo o norte da América do Sul, ao centro da floresta amazônica. A presença de usinas de beneficiamento, como a Usina de Boca do Acre no Amazonas e Usina Xapurí no Acre que também são determinantes para a produção desses PFNMs, de grande escala produtiva na região da Amazônia Legal. Além disso, o Acre conta com o planejamento das práticas de manejo desenvolvidas pela Embrapa na reserva Chico Mendes, que priorizam a sustentabilidade ambiental e a potencialidade da floresta em continuar fornecendo recursos naturais.

Os municípios que constituem o cluster 3 pertencem em sua maioria ao estado do Maranhão, com alguns poucos pertencentes aos Estados do Mato Grosso, Amazonas, Acre e Tocantins. São caracterizados pela maior produtividade de amêndoas, especificadamente Babuçu e Pequi, e tanante angico. Segundo Albieiro et al (2007) os estados do Maranhão e Tocantins concentram as maiores extensões de matas onde predominam os babaçus, formando, muitas vezes e espontaneamente, agrupamentos homogêneos, bastante densos e escuros, tal a 
proximidade entre os grandes coqueiros. Só no Maranhão a disponibilidade de biomassa anual alcança cerca de 1,5 milhões de toneladas e em Tocantins 28 mil toneladas (Teixeira, 2002). Apesar de sua exploração estar baseada num extrativismo primário, o babaçu desempenhou até meados da década de 80, importante papel na economia, principalmente, do Estado do Maranhão, como base da sustentação de um parque industrial de extração de óleo vegetal (comestível e biodiesel), instalado exclusivamente para processar as amêndoas oleaginosas extraídas do seu fruto. Segundo Albieiro et al (2007).

"A venda de amêndoas de babaçu representa para os pecuaristas receitas brutas de US\$18.40/ha que em receitas líquidas representa US\$ 4.60/ha por ano. Embora estas receitas pareçam baixas, quando comparadas com a renda proveniente da pecuária (US\$ 15.45) representam uma renda adicional de quase um quarto dos rendimentos líquidos por hectare provenientes de ambas as atividades. O babaçu além de ser uma fonte importante de renda para as populações locais que o extraem, o é também para a economia regional como um todo através da industrialização do óleo de babaçu que na década dos 80 atingiu uma produção de 80.000 toneladas/ano, o que gerou um valor no mercado final de 40 milhões de dólares”. (Albieiro et al , 2007, pg. 15).

A produtividade alta de babaçu dos municípios do cluster C3 se deve, portanto a sua disponibilidade natural. A principal importância desse produto esta relacionada ao sustento dos extrativistas, já que o babaçu é produzido pela população extremamente pobre da região amazônica por absoluta falta de outras oportunidades. Eles extraem os caroços do fruto lenhoso que é um trabalho extremamente penoso. Os caroços são vendidos às fabricas para a extração do óleo ou são transformadas em óleo de forma caseira para o consumo da família (Clement, 2005).

Por último, analisando a distribuição dos municípios do cluster C4 que são caracterizados por produzirem todos os 21 PFNMs selecionados, nota-se a partir do Mapa 02 que eles estão localizados na região centro-norte da Amazônia, mais especificadamente, constituem muitos municípios dos estados do Pará, Amapá, Acre, Amazonas e Rondônia. O que significa que esses estados possuem uma diversidade grande em relação a produtividade de produtos florestais não madeireiros. Tal fator pode estar atrelado ao fato de grande parte desses municípios não possuir áreas naturais protegidas capazes de limitar o avanço do extrativismo predatório, onde a exploração é realizada livremente, e por pertencerem a alguns estados mais ricos economicamente da região Amazônica como Pará e Amazonas, onde são incentivados tecnologias e melhoramentos para a produção de PFNMs e infraestrutura de beneficiamento e escoamento o que mantém a manutenção da produtividade e a geração de empregos e renda para os extrativistas e pequenos produtores. Além disso, é preciso levar também em consideração a disponibilidade natural desses produtos nas regiões de floresta desses municípios. 


\section{CONSIDERAÇÕES FINAIS}

A Amazônia ainda é detentora de recursos naturais abundantes e disponíveis que são utilizados sem a preocupação de sua conservação para as gerações futuras. Uma das principais preocupações relacionadas ao incentivo da produção dos PFNMs é conciliar uma maior rentabilidade e qualidade de vida para as famílias dependentes do extrativismo com um manejo florestal sustentável.

Nesse contexto faz-se necessário não só a criação de políticas públicas, mas também muitos investimentos direcionados para produção de PFNMs, já que um dos grandes problemas encontrados na região é a falta de planejamento, tecnologias e técnicas adequadas para o sistema de produtivo. Bentes (2005) afirma que a dificuldade de acesso às áreas onde os PFNM são abundantes, particularmente na Amazônia, é também um dos entraves quemantêm a retirada a granel destes produtos, colocados imediatamente à venda sem maior possibilidade de agregação de valor, devido ao alto custo de transporte até os pontos de comercialização dos centros consumidores. Alguns estudiosos (Tonini ) já alertavam para esta realidade e destacaram que qualquer PFNM a ser comercializado além de ter que apresentar um alto valor agregado, deve ocorrer de modo uniforme no bioma regional e ter um atrativo especial, como por exemplo, estar relacionado a uma estratégia de desenvolvimento sustentável para o local onde ocorre.

Se tratando dessas ocorrências os resultados de produtividade agregados por municípios através do algoritmo FUNNY demonstraram que apenas 35\% dos municípios (cluster C1) não apresentaram produção de PFNMs e $32 \%$ apresentaram produção de todos os PFNMsselecionados entre os anos de 2004 a 2008. Segundo o IBGE (2009) A Amazônia legal apresentou em 2008, 92,2\% da produção nacional de açaí (fruto), 95,4\% da produção de castanha-do-pará e 12,4\% da produção de fibras de piaçava. Os dados demonstram, portanto, o grande potencial da floresta e a possível criação de um mercado mais sólido para esses produtos a partir dos incentivos de produção.

Tendo em vista a espacialidade dos clusters/grupos de município, pode-se concluir que uma regionalização em torno da produção de PFNMs classificaria a Amazônia em quatros subregiões características. Uma sub-região seria no centro-norte da Amazônia, caracterizada pela alta produtividade de castanha e borracha, o estado do Pará formaria outra sub-região pela sua hegemonia na produtividade do açaí. Outra sub-região seria parte dos municípios localizados a leste do estado do Maranhão e parte dos municípios localizados ao norte de Tocantins, pela hegemonia e alta produtividade de Babaçu, por último os estado do Mato Grosso e os restantes dos municípios dos estados do Maranhão e Tocantins pela ausência de produtividade. 
Para uma melhor analise espacial, uma posposta futura de aprimoramento desse trabalho seria aplicar aos dados a função Média Espacial Móvel que se contitui em uma forma simples e útil de explorar a variação da tendência espacial dos dados, calculando a média dos valores dos vizinhos. Isto produz uma primeira aproximação da variabilidade espacial, pois a operação tende a produzir uma superfície menos descontínua (mais suave) que os dados originais, podendo ainda apresentar indicações de locais de transição entre regimes espaciais.

\section{REFERÊNCIAS BIBLIOGRÀFICAS}

ALBIERO. Daniel;MACIEL.Antonio.Proposta de uma máquina para colheita mecanizada de babaçu (Orbignyaphalerata Mart.) para a agricultura familiar. Acta Amaz. vol.37 no.3.Manaus 2007.

BRITO, J. O. Produtos florestais não-madeireiros: um importante potencial nas florestas. Boletim Informativo ARESB, Avaré, n. 47, p.4, 2003.

BENTES. Michellyni. Principais relações de comercialização de produtos florestais não madereiros (pfnm) na Amazônia. Embrapa, 2005. Disponível em: www.agronline.com.br (Acessado em 22/11/2010)

CASTRO. Dulcilene. A. Práticas e técnicas com produtos florestais não madeireiros: Um estudo de caso com famílias no pólo rio capim do proambiente. Amazônia: Ci. \&Desenv., Belém, v. 2, n. 4, jan./jun. 2007. 
CASTELLANI, D. C. Plantas medicinais e aromáticas: produtos florestais não madeireiros (PFNM). In: SEMINÁRIO MATOGROSSENSE DE ETNOBIOLOGIA E ETNOECOLOGIA; SEMINÁRIO CENTRO-OESTE DE PLANTAS MEDICINAIS. Cuiábá, 2002)

EMBRAPA - EMPRESA BRASILEIRA DE PESQUISA AGROPECUÁRIA. Manejo florestal não madeireiro em unidade de conservação de uso direto. Rio Branco: EMBRAPA, 2000. 4p. (Folheto).Disponivel em: WWW.embrapa.br (Acessado em 22/11/2010)

FILDER. Nilton César. Produtos Florestais Não Madeireiros: Importância e Manejo Sustentável da Floresta. Revista Ciências Exatas e Naturais, Vol.10 nº 2, Jul/Dez 2008.

KAUFMAN, L. \& ROUSSEEUW, P.J. Finding groups in data: an introduction to cluster analysis. New York: Wiley, 1990.

Portal Ministério da Saúde. Disponível: www.saude.gov.br. (Acessado em 22/11/2010)

PORTAL DO EXTRATIVISMO. Castanha do Brasil e Açai. Disponível em: www.portaldoextrativismo.com.br. (Acessado em 22/11/2010)

REVISTA ONLINE. Noticias da Amazônia. Disponível em: www.noticiasamazonia.com.br (Acessado em 22/11/2010)

RIBEIRO, A. M.; GARCIA, R. A. Segregação socioespacial em Belo Horizonte: uma aplicação de modelos difusos. Cedeplar/UFMG, Belo Horizonte, 2005

SIMÕES, Rodrigo. COMPLEXOS INDUSTRIAIS NO ESPAÇO: UMA ANÁLISE DE FUZZY CLUSTER. Anais do XXXII Encontro Nacional de Economia.Cedeplar. Belo Horizonte. 2004

SIDRA. Sistema do IBGE de Recuperação Automática. Dados de produção agrícola municipal 2009. Disponível em: www.sidra.ibge.br. (Acessado em 22/11/2010)

TEIXEIRA. Marcus. Biomassa de babaçu no Brasil. An. 4. Enc. Energ. Meio Rural 2002. Disponivel em: www.scielo.com.br (Acessado em 22/11/2010)

TONINI Helio; COSTA Patrícia, KAMINSKI. Manejo de Produtos Florestais Não Madeireiros na Amazônia - (Castanheira-do-Brasil). Resultados de pesquisa.Embrapa. Boa Vista. 2008. 